\title{
DISTRITOS INDUSTRIAIS E INOVAÇÃO: O SECTOR DOS MOLDES EM PORTUGAL
}

\author{
Filipa D. Vieira* ${ }^{1}$, Fernando Romero ${ }^{2}$ \\ ${ }^{1}$ Universidade do Minho, Depart. Produção e Sistemas (DPS) - Guimarães, Portugal \\ ${ }^{2}$ Universidade do Minho, Depart. Produção e Sistemas (DPS) - Guimarães, Portugal \\ *Email: filipadv@dps.uminho.pt
}

\section{RESUMO}

Com este estudo pretendeu determinar-se onde e como oito empresas nacionais do sector dos moldes adquirem o conhecimento necessário para as suas actividades de inovação. A ênfase deste trabalho foi o estudo das interacções entre vários elementos do sistema, sejam eles internos ou externos à empresa. A capacidade inovadora do sector é também relacionada com certas características intrínsecas do mesmo.

$\mathrm{Na}$ verdade, pode afirmar-se que este sector da indústria nacional possui algumas características idênticas à de um distrito industrial italiano, e este facto influenciou e condicionou o seu desempenho inovador.

\section{INTRODUÇÃO}

Com base no estudo de oito empresas nacionais do sector dos moldes, todas localizadas na região da Marinha Grande, pretendeu determinar-se onde e como tais empresas adquirem o conhecimento necessário para a realização das suas actividades e desempenho inovadores. Interessa saber se tais conhecimentos foram adquiridos interna e/ou externamente através de redes de inovação e determinar quais os principais elementos que constituem tais redes, bem como qual o seu desempenho, como impulsionadores da inovação. A ênfase deste trabalho está no estudo das interacções entre os vários elementos do sistema, sejam eles internos ou externos à empresa. Segundo Lundvall (1992), o conhecimento é o recurso mais importante na moderna economia e, consequentemente, a aprendizagem é o processo mais importante, sendo este predominantemente interactivo e decorre num enquadramento ou contexto institucional e cultural. A aprendizagem está relacionada com actividades de rotina, em diferentes áreas - na produção, na distribuição ou no consumo - permitindo, assim, a criação de importantes inputs para o processo de inovação.

O estudo efectuado resultou na criação de um modelo de observação do sector dos moldes, relativo ao tipo de relações existentes entre as empresas e entre estas e outras instituições, permitindo uma sistematização e uma generalização na análise da dinâmica inovadora deste sector.

A capacidade inovadora do sector é também relacionada com certas características intrínsecas do mesmo. Ao estudar as empresas, bem como as interacções existentes, constatou-se a existência de características particulares dos distritos industriais italianos. Evidenciam-se os factos de: (1) haver uma concentração geográfica de pequenas e médias empresas; (2) as empresas não só cooperarem entre si, devido à sua proximidade geográfica e à sua envolvente sócio-cultural, mas também concorrerem e se relacionarem mais ou menos intensivamente 
com todos os outros actores (fornecedores, clientes, consultores e instituições de saber); (3) haver especialização numa ou em várias fases do processo produtivo, estando um determinado produto associado à região, que a passou a identificar; (4) a produção ser flexível, indo ao encontro das necessidades dos clientes e (5) existir empresas líderes que facilitam a expansão internacional da região.

\section{REVISÃO BIBLIOGRÁFICA}

É amplamente assumido que a inovação é um factor-chave na competitividade e que o crescimento do output e da produtividade dependem do desenvolvimento e da difusão de novas tecnologias (OCDE, 1997). Daí urge a necessidade de as empresas apostarem fortemente na inovação, porque, só assim, conseguirão crescer com mais conhecimento e produzir com mais valor acrescentado, tornando-se mais competitivas.

Contudo, o processo de inovação não é linear, mas sim um processo complexo, interactivo, envolvendo instituições empresariais e não empresariais. $\mathrm{O}$ modelo de ligação em cadeia ou "chain-linked model", apresentado por Kline e Rosenberg (1986) é o exemplo de um modelo de inovação que mostra o carácter interactivo do processo de inovação. Na verdade, a inovação não deve ser vista como o produto de um único actor, mas sim o resultado da interacção entre vários actores, quer institucionais quer organizacionais. E apesar do processo de inovação ser descontínuo e incerto, os sistemas nacionais de inovação ajudam a perceber e explicam porque é que a tecnologia se desenvolve numa determinada direcção.

O estudo de sistemas nacionais de inovação consiste na análise das ligações existentes entre as várias partes que compõem o sistema de inovação, sejam elas as empresas, as instituições académicas ou as instituições de investigação, públicas ou privadas (Nelson, 1993; Lundvall, 1992; Freeman, 1987). Não esquecendo que são as empresas o 'core' destes sistemas, competindo e, simultaneamente, cooperando umas com as outras (Dosi et al., 1988).

Diversos autores (Chung and Kim, 2003; Freel, 2003; Beeby and Booth, 2000; Sousa, 1997) referem a importância das interacções entre empresas e o ambiente social e económico onde estas se encontram inseridas, resultando assim em ligações com clientes e fornecedores, com empresas de consultadoria e com infra-estruturas da ciência e tecnologia, salientando a importância dos centros de I\&D e das instituições de educação e formação. A optimização destas ligações e das competências associadas resultam no estabelecimento de redes (de inovação e de negócio) que assentam na complementaridade de competências ou de activos, na dependência mútua e em novas formas de organização económica, não primordialmente regidas por vínculos contratuais mas principalmente por relações de confiança e de partilha de riscos e benefícios (Teece, 1997).

Empresas que apresentam altos níveis de colaboração tecnológica, quer entre empresas, quer com universidades e institutos de investigação públicos, e de aquisição de tecnologia e mobilidade de recursos humanos, conseguem melhorar a sua capacidade inovadora em termos de produtos e processos. A facilidade com que o conhecimento é transferido depende do seu tipo. O conhecimento explícito é formal e sistemático, portanto pode estar facilmente associado a especificações de produtos, fórmulas ou programas de computador. $\mathrm{O}$ conhecimento tácito é, em parte, capacidade técnica, mas também tem uma dimensão cognitiva formada por modelos mentais, crenças e perspectivas, que são difíceis de articular. A socialização é a única maneira de transferir conhecimento tácito entre indivíduos, através da observação, imitação e prática (Arias, 1995). 
Talvez por isso, as regiões parecem ter importância acentuada para o desenvolvimento de redes e de sistemas de novas tecnologias. As infra-estruturas locais, os recursos humanos especializados, o mercado de trabalho local, os serviços especializados e as relações pessoais são fundamentais e contribuem para o desenvolvimento regional (Muscio, 2006; Chung, 2002; Freeman e Soete, 1997).

Quando se fala em regiões, rapidamente se associa a conceitos como cluster (Porter, 1993; Porter, 1998) ou cluster geográfico de pequenas e médias empresas (Carbonara, 2005; Piscitello e Sgobbi, 2004), também definido como distrito industrial (Becattini, 1990). Os clusters são vistos como uma oportunidade estratégica para as pequenas e médias empresas prosperarem em determinados sectores da indústria, porque as empresas localizadas em clusters parecem ser potencialmente mais inovadoras do que as não localizadas, que resulta do facto de estarem próximas, quer geograficamente quer estrategicamente, facilitando as trocas de conhecimento entre as empresas e entre estas e outras instituições (Staber, 2001a; Porter, 1998).

Os distritos industriais são definidos na literatura como sistemas de produção geograficamente concentrados, constituídos por um número elevado de pequenas e médias empresas, que estão envolvidas em várias fases de produção de um mesmo produto ou família de produto. Estas empresas são altamente especializadas em algumas fases do processo de produção, sendo a sua integração feita através de ligações e cooperações entre essas mesmas empresas (Albino et al., 2006; Molina-Morales e Martínez-Fernández, 2004a; Piscitello e Sgobbi, 2004; Guerrieri e Pietrobelli, 2004; Chiarvesio et al., 2004; Corolleur e Courlet, 2003; Giner e Santa Maria, 2002; Sammarra e Biggiero, 2001; Becattini, 1990; Marshall, 1988).

Partindo da definição de distrito industrial de Becattini e com base em vários trabalhos (Molina-Morales, 2005; Piscitello e Sgobbi, 2004; Carbonara, 2002; Giner e Santa Maria, 2002; Sammarra e Biggiero, 2001; Day et al. 2000; Rabellotti, 1995; Pyke e Sengenberger, 1992; Becattini, 1990; Capecchi, 1990; Piore, 1990; Pyke e Sengenberger, 1990; Marshall, 1988; Piore e Sabel, 1984) é possível enumerar um conjunto de factores que o caracterizam:

1. Existem muitas pequenas e médias empresas num determinado território, mas com o mesmo tipo de produção flexível;

2. Existência de fortes ligações de cooperação entre as empresas, baseadas na confiança;

3. Além de existir ligações entre as empresas, também existem relações entre estas e as instituições locais, relacionando-se estas últimas com outras empresas e outras instituições localizadas fora do distrito. $\mathrm{O}$ que facilita a criação de valor às empresas pertencentes ao distrito;

4. As empresas tendem a especializar-se numa ou em várias fases do processo produtivo, contribuindo assim para uma mesma produção, quer seja de produtos finais quer seja de produtos intermédios, resultando na divisão de trabalho entre as empresas. A cada distrito industrial está associado um produto, que passará a ser característico desse mesmo distrito industrial;

5. A produção é flexível e tenta ir ao encontro das necessidades dos clientes. As empresas têm capacidade de produzir sistemas de produtos; 
6. Independentemente da dimensão das empresas, algumas produzem/vendem directamente para o cliente final (mercado), outras produzem apenas partes do produto ou fazem apenas parte de uma fase do processo;

7. A divisão entre empresas que vendem directamente os seus produtos e as que actuam como subcontratadas por outras empresas não é rígida, isto é, uma pequena empresa pode funcionar em determinado período como uma subcontratada e noutro como uma vendedora;

8. As relações entre empresas que vendem directamente para o mercado são ao mesmo tempo de competição e de cooperação; o que significa que as empresas não entram em conflito umas com as outras, mas tentam encontrar novos mercados, evitando criar um efeito destrutivo para o próprio distrito industrial;

9. A área é bem definida porque diz respeito a uma área geográfica bem delimitada, sendo especificamente caracterizada pelo domínio de determinada produção;

10. Existe uma forte relação dentro do distrito, nomeadamente entre a realidade produtiva e a envolvente sócio-económica, havendo de certa forma uma fusão entre as empresas e a comunidade, graças à sua homogeneidade de valores, sendo este um requerimento essencial para o desenvolvimento dos distritos industriais;

11. A existência de empreendedores puros nos distritos industriais é bastante frequente. Estes agem como seguidores do que melhor se faz internacionalmente, relativamente à produção e aos produtos, com o objectivo de melhorar o que já se faz no distrito, utilizando para isso o conhecimento acumulado para produzir novos produtos;

12. A presença de empresas líderes que ajudam à expansão internacional do distrito industrial;

13. A mobilidade dos recursos humanos, característica extremamente importante, porque o conhecimento está nas pessoas (o know-how passa de geração em geração) e não nas empresas. $\mathrm{O}$ que permite a difusão de conhecimento pelas empresas do distrito industrial. A grande mobilidade dos recursos humanos contribuiu também para o processo de aprendizagem colectivo.

Aos distritos industriais está, principalmente, associada a existência de ligações entre os diferentes actores que o constituem, nomeadamente as empresas, as instituições locais (instituições de ensino, de investigação, de formação), as empresas líderes e as empresas especializadas $\left(\mathrm{KIBS}^{1}\right)$, que permitem uma troca e combinação de recursos e conhecimento. Molina-Morales e Martínez-Fernández (2004b) argumentam que os distritos industriais permitem a partilha de recursos, o que explica a superioridade competitiva das empresas que os constituem e que se deve ao facto de: (1) terem a mesma reputação; (2) existir uma intensa troca e combinação de recursos e (3) haver a participação de instituições locais.

\footnotetext{
${ }^{1}$ As KIBS (Knowledge Intensive Business Services) são empresas especializadas em determinados serviços e prestam serviço a outras empresas, evidenciando-se em áreas do conhecimento, como por exemplo nas áreas do design (Muller and Zenker, 2001)
} 


\section{MODELO DE OBSERVAÇÃO E ANÁLISE DE EMPRESAS INOVADORAS DO SECTOR DOS MOLDES}

Apesar de todas as empresas estudadas pertencerem ao mesmo sector da indústria nacional Sector dos Moldes -, as empresas apresentam competências diferentes, o que permite agrupálas segundo a sua posição na cadeia de valor do mesmo, criando uma tipologia específica para cada um desses grupos de empresas, como se pode constatar pela análise da figura 1, abaixo apresentada.

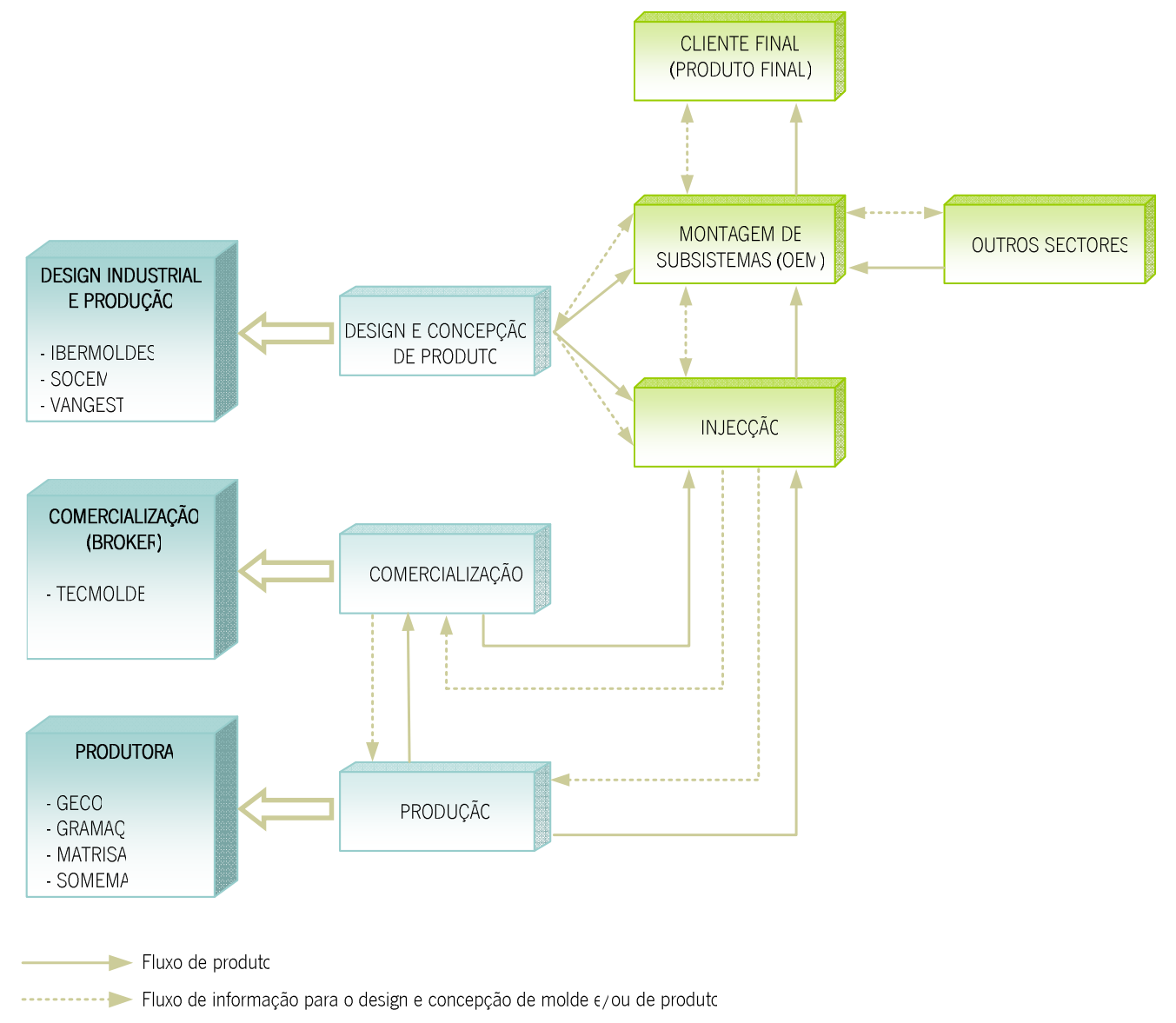

Figura 1: Representação da cadeia de valor do sector dos moldes e sua relação com a tipologia das empresas estudadas

Da observação da figura 1 ressalta: (1) a cadeia de valor do sector assenta em várias actividades, que podem ir desde a produção até à entrega do produto final ao cliente final, evidenciando-se o fluxo de produto e o fluxo de informação para o design e concepção de molde e/ou produto; (2) as áreas nucleares de competências das empresas estudadas que permitiram a criação de uma tipologia de empresas: produção, comercialização e design e concepção de produto e (3) as diferentes empresas de moldes estudadas associadas à tipologia, entretanto criada: GECO, GRAMAQ, MATRISA e SOMEMA (Produtora); 
TECMOLDE (Comercialização) e IBEROMOLDES, SOCEM e VANGEST (Design Industrial e Produção).

A tabela 1, a seguir apresentada, foi construída a partir da síntese dos resultados do estudo realizado e propõe um modelo conceptual de observação das empresas inovadoras do sector dos moldes, mostrando como as características das empresas estudadas, que dependem da sua posição na cadeia de valor do sector em questão, se reflectem em termos das relações existentes com outras empresas, tais como clientes, fornecedores, concorrentes e com instituições, sejam elas, de ensino superior, de investigação públicas e privadas e infraestruturas tecnológicas. Permite, ainda, tirar ilações sobre as áreas nucleares de competências, a origem das ideias para o desenvolvimento das inovações e o tipo de inovações que são desenvolvidas pelas empresas. Sendo assim, as empresas comportam-se, em termos inovadores, de maneiras distintas, estando fortemente dependentes do padrão de ligações informais que mantêm. 
Tabela 1: Modelo de observação do sector dos moldes em Portugal

\begin{tabular}{|c|c|c|c|c|c|c|c|c|c|c|c|}
\hline Tipo de empresa & $\begin{array}{l}\text { Área nuclear de } \\
\text { competências }\end{array}$ & $\begin{array}{l}\text { Origem das } \\
\text { ideias }\end{array}$ & Tipo de inovação & $\begin{array}{c}\text { Dependência dos } \\
\text { clientes para o } \\
\text { desenvolvimento de } \\
\text { inovações } \\
\end{array}$ & & $\begin{array}{c}\text { Relação com } \\
\text { fornecedores de: }\end{array}$ & & $\begin{array}{l}\text { Relação com } \\
\text { concorrentes }^{2}\end{array}$ & $\begin{array}{l}\text { Relação com } \\
\text { instituições } \\
\text { académicas }\end{array}$ & $\begin{array}{l}\text { Relação com } \\
\text { centros de } \\
\text { investigação }\end{array}$ & $\begin{array}{l}\text { Relação com } \\
\text { centros de } \\
\text { formação }\end{array}$ \\
\hline & & & & & Materiais $^{4}$ & $\begin{array}{l}\text { Máquinas e } \\
\text { equipamentos }\end{array}$ & Software & & & & \\
\hline $\begin{array}{l}\text { Produtora } \\
\text { - GECO } \\
\text { - GRAMAQ } \\
\text { - MATRISA } \\
\text { - SOMEMA }\end{array}$ & Produção & Externa & Processo & Forte & Fraca & Forte & Forte & Forte & $\begin{array}{l}\text { Fraca ou } \\
\text { inexistente }\end{array}$ & $\begin{array}{l}\text { Fraca ou } \\
\text { inexistente }\end{array}$ & Forte \\
\hline $\begin{array}{l}\text { Comercial (Broker) } \\
\text { - TECMOLDE }\end{array}$ & Comercialização & Externa & $\begin{array}{l}\text { Processo e } \\
\text { Produto }\end{array}$ & Forte & Forte & Forte & Forte & Inexistente & Inexistente & Inexistente & Inexistente \\
\hline $\begin{array}{l}\text { Design Industrial } \\
\text { e Produção } \\
\text { - IBEROMOLDES } \\
\text { - SOCEM } \\
\text { - VANGEST }\end{array}$ & $\begin{array}{c}\text { Design e } \\
\text { concepção de } \\
\text { produto }\end{array}$ & Interna & $\begin{array}{l}\text { Processo e } \\
\text { Produto }\end{array}$ & Fraca & Médio & Forte & Forte & Forte & Forte $^{5}$ & Forte & Fraca \\
\hline
\end{tabular}

\footnotetext{
${ }^{2}$ Estas relações só ocorrem com alguns concorrentes e esta classificação foi feita com base neles

${ }^{3}$ Refere-se ao CENTIMFE e ao INETI

${ }^{4}$ Refere-se principalmente aos fornecedores de aço

${ }^{5}$ Pode considerar-se forte quando se compara com os restantes grupos, uma vez que nestes as relações existentes são quase nulas, mas continua a evidenciar-se um grande afastamento entre estas duas entidades, devido, acima de tudo, a barreiras culturais que estão a ser transpostas, ainda de forma muito tímida
} 


\subsection{Empresas Produtoras}

As empresas que são produtoras por excelência têm uma elevada preponderância para desenvolverem inovações de processo, que advêm da sua forte dependência dos clientes em termos de concepção do molde que é, em geral, desenvolvido externamente, pelo cliente.

Neste tipo de empresas, as competências internas necessárias para a geração de ideias para a inovação de produto são escassas. A origem das ideias para a inovação centra-se, principalmente, nos clientes e, pontualmente, nos fornecedores de máquinas e de equipamentos. $\mathrm{Na}$ verdade, são as exigências dos clientes que obrigam, por seu turno, ao desenvolvimento do processo produtivo. Os clientes são os grandes impulsionadores da aquisição de novos equipamentos que permitem, através de novas e melhoradas técnicas de produção, a satisfação dos requisitos exigidos.

As relações com fornecedores de materiais (nomeadamente os fornecedores de aço) são muito reduzidas, não havendo neles uma fonte de informação significativa para a inovação. Contudo, as relações são consistentes com as empresas fornecedoras de máquinas, equipamentos e software.

Neste tipo de empresas, o relacionamento com centros de investigação é ténue ou mesmo inexistente, principalmente quando se trata de universidades, mas vão mantendo algumas relações com o CENTIMFE, nomeadamente na obtenção de alguma formação.

Estas empresas são típicas empresas subcontratadas, que vão evoluindo tecnologicamente em função das exigências dos seus clientes e do conhecimento transferido pelos fornecedores de máquinas e de equipamentos.

\subsection{Empresa Comercial (Broker)}

Esta empresa funciona como um broker, uma vez que tem um comportamento misto relativamente aos outros dois grupos identificados (Produtora e Design Industrial e Produção). É uma empresa que não possui capacidade produtiva própria, subcontratando toda a sua produção a empresas exclusivamente produtoras (a primeira categoria), bem como àquelas de serviços especializados. Possui competências ao nível da engenharia e concepção do molde e muito conhecimento sobre o mercado, daí apresentar um elevado desempenho comercial. Face ao exposto, as suas características encontram-se entre as duas situações "extremas".

Este tipo de empresa é fulcral para a sobrevivência de muitas empresas do sector dos moldes, porque assegura duas funções que se revelam essenciais para muitas empresas. A primeira é uma função comercial, de ligação ao mercado externo, que é inexistente em muitas empresas do sector e, através desta função, assegura o escoamento e a exportação de uma parte substancial da produção de muitas empresas. A segunda função prende-se com a gestão de carteiras de encomendas ${ }^{6}$ e a potenciação de economias de escala, através da coordenação da actividade produtiva de um conjunto de empresas. Esta vertente advém da incapacidade de resposta da maior parte das empresas a grandes encomendas e da necessidade de distribuir a produção da encomenda por várias empresas, de maneira a cumprir os prazos de entrega. Assim, esta empresa desenvolve capacidades de coordenação e de integração, ao distribuir produção e capacidade por uma rede de empresas produtoras, criando um formato organizacional que é equivalente aos propostos pelos conceitos de empresa "estendida" ou "virtual" (Browne e Zhang, 1999).

\footnotetext{
${ }^{6}$ A tendência do mercado passa cada vez mais pela procura de sistemas de moldes e não tanto de moldes isolados
} 


\subsection{Empresas com Design Industrial e Produção}

As empresas deste grupo têm a componente de engenharia e concepção de moldes bastante desenvolvida, conjuntamente com a parte produtiva, além de possuírem competências emergentes no design e na concepção de produtos injectados, sendo, assim, capazes de fornecer soluções inovadoras aos seus clientes. A sua dependência dos clientes para o desenvolvimento de inovações é fraca, pois o seu know-how é suficientemente elevado para fornecer, autonomamente, as soluções que os clientes procuram.

Estas empresas mantêm relações relativamente consistentes com as instituições de educação e de investigação, apesar de considerarem que essas ligações deveriam ser mais frequentes. Recorrem com alguma regularidade às universidades, aos laboratórios de investigação e ao centro tecnológico, funcionando estes como parceiros privilegiados na resolução dos seus problemas técnicos. Como consequência deste comportamento, são empresas em que a realização de inovações de produto se torna mais importante, sem prejudicar a importância das inovações de processo. O know-how entretanto adquirido permite-lhes inovar já em termos de produto, adoptando uma estratégia de diversificação mais acentuada.

\section{ANALOGIA ENTRE O SECTOR DOS MOLDES DA MARINHA GRANDE E OS DISTRITOS INDUSTRIAIS ITALIANOS}

A análise detalhada das relações existentes entre as empresas de moldes e os seus parceiros (outras empresas e instituições) e a importância de tais ligações para o desempenho inovador das empresas em particular e do sector em geral levou a uma possível comparação com os distritos industriais italianos. Os distritos industriais são uma forma particular de organização industrial, sendo largamente reconhecido a sua importância, no desenvolvimento de sectores tradicionais em Itália. Contudo, não é só em Itália que existe este tipo de organização industrial, existindo também noutros países, sempre associado a sectores tradicionais, como por exemplo a cerâmica em Espanha (Castelón) (MolinaMorales, 2002) e a têxtil na Alemanha (Baden-Wurttemberg) (Staber, 2001b).

Com base na realidade industrial da região da Marinha Grande e tomando como referência os distritos industriais italianos é possível observar algumas semelhanças entre os dois. Nomeadamente a presença de um número elevado de pequenas e médias empresas, localizadas numa região bem definida, que apresentam níveis de especialização e flexibilidade consideráveis e pertencentes a um sector considerado tradicional da indústria nacional. O sector dos moldes localizado na região da Marinha Grande cresceu rapidamente, aparecendo novas empresas a partir de spin-offs de outras empresas já existentes. São significativas as relações de competição entre as empresas, bem como as relações de cooperação, que são principalmente informais. Como existe algum nível de especialização das empresas, o recurso à subcontratação é corrente neste sector da indústria. A utilização de recursos disponíveis na região também é frequente, apesar de muitos dos recursos necessários ao processo de fabricação não existirem, mas existem instituições locais e empresas especializadas que servem de apoio às empresas do sector. A existência de empresas líderes é outra característica importante dos distritos industriais italianos e presente no sector dos moldes da região da Marinha Grande. Tais empresas são essenciais ao desenvolvimento e melhoria do desempenho das outras empresas do sector, aumentando a sua competitividade, porque difundem conhecimento e promovem a sua expansão.

Apesar do sector dos moldes da Marinha Grande apresentar algumas características semelhantes à dos distritos industriais italianos, a intensidade com que ocorrem é bem distinta, particularmente no que diz respeito às relações de cooperação entre as empresas de moldes concorrentes, que ocorrem apenas entre aquelas, em que as relações pessoais e a confiança entre os dirigentes são significativas. $\mathrm{O}$ 
número de empresas subcontratadas não é tão elevado quanto o caso italiano, porque a divisão do trabalho não é tão acentuada, devendo-se ao facto do nível de especialização nas diferentes fases do processo de fabricação de moldes ser mais reduzido quando comparado com o caso italiano. As empresas de moldes localizadas na região da Marinha Grande tanto subcontratam fases do processo como todo o processo produtivo.

Como já foi referido, uma outra característica relevante no estudo de distritos industriais italianos é a utilização por parte das empresas dos recursos disponíveis internamente, necessários à fabricação do produto que identifica tal distrito industrial. No caso particular do sector dos moldes da região da Marinha Grande constata-se que a utilização de tais recursos é bastante inferior quando comparado com o caso italiano. As razões prendem-se, principalmente, com o facto de não existirem alguns desses recursos na região e com a pouca utilização dos existentes pelas empresas do sector dos moldes aí localizadas. Relativamente à não existência de alguns recursos, refere-se em particular os fornecedores de máquinas e equipamentos, onde toda a tecnologia é importada.

Outra particularidade desta região é o facto dos clientes se encontrarem fora da região. Este sector da indústria nacional é essencialmente exportador, o mercado interno é pouco representativo, portanto também a procura se localiza fora do espaço geográfico desta região, situação bem diferente de alguns distritos industriais italianos, em que a procura se localiza no próprio distrito. $\mathrm{O}$ grupo de empresas referido na secção anterior, que foi classificado sob o título de brokers, baseia a sua actividade no aproveitamento desta característica, servindo de ponte entre o cliente final (no estrangeiro) e a empresa produtora. Mas, apesar da procura ser essencialmente externa, a maioria das empresas locais mais importantes mantêm fortes e consistentes relações com os seus clientes, tendo tais ligações sido uma importante fonte de informação, grandemente responsável pelo desempenho deste sector. Tratase de clientes pertencentes a sectores da indústria muito exigentes, como por exemplo o sector automóvel, que têm estimulado as empresas do sector dos moldes a melhorar continuamente, tornando-as em fortes concorrentes em termos internacionais.

O facto de o sector dos moldes da Marinha Grande, não possuir alguns elementos característicos dos distritos industriais italianos, como a disponibilidade interna de alguns recursos, nomeadamente os fornecedores de materiais e de tecnologia, e não ter procura interna, não invalida os pressupostos e as conclusões acima mencionados. Alguns autores (Chiarvesio et al., 2004; Staber, 2001a) defendem que a proximidade geográfica de empresas começa a ser menos importante para o desenvolvimento de actividades de inovação por parte das empresas e consequentemente para o respectivo desenvolvimento da região, onde tais empresas estão inseridas, i.e., a proximidade geográfica como factor de vantagem competitiva começa a ser questionada, devido ao aparecimento de novas tecnologias de informação. E (Lazerson e Lorenzoni, 1999, p. 370) acrescentam, ainda, que "actores fora do distrito industrial são muitas vezes indispensáveis, porque actuam como agentes que espalham as sementes de futuro desenvolvimento".

\section{CONCLUSÕES}

Pode-se assim concluir, que a grande vantagem competitiva deste sector da indústria nacional se deve à sua forte capacidade de se relacionar com outros parceiros, independentemente da sua posição na cadeia de valor. Mas estas ligações são diferenciadas, tanto em termos de parceiros como em termos de intensidade, verificando-se uma correlação entre a capacidade e o tipo de actividade inovadora e as interacções estabelecidas pela empresa.

O facto de o sector dos moldes, localizado na região da Marinha Grande, apresentar características idênticas às de um distrito industrial italiano, influenciou o seu desempenho inovador e aumentou a sua competitividade. Esta forma de organização industrial facilita a inovação e a difusão de inovações, factor crítico para obtenção de competitividade por parte de pequenas e médias empresas, devido à 
dificuldade na prossecução de actividades de investigação e desenvolvimento, associada à falta de recursos financeiros e humanos qualificados e ao risco elevado.

\section{REFERÊNCIAS}

- Albino, V.; Carbonara, N.; Giannoccaro, I., Innovation in industrial districts: An agent-based simulation model, International Journal of Production Economics, Vol. 104, No 1, 2006

- Arias, J. T. G., Do networks really foster innovation?, Management Decision, Vol. 33, № 9, 1995

- Beeby, M.; Booth, C., Networks and inter-organizational learning: A critical review, The Learning Organization, Vol. 7, No 2, 2000

- Becattini, G., The Marshallian industrial district as a socio-economic notion, in: Pyke, F.; Becattini, G.; Sengenberger, W. (eds.), Industrial districts and inter-firm co-operation in Italy, International Institute of Labour Studies, Geneva 1990

- Browne, J.; Zhang, J., Extended and virtual enterprises - Similarities and differences, International Journal of Agile Management Systems, Vol.1, N 1, 1999

- Capecchi, V., A history of flexible specialisation and industrial districts in Emilia-Romagna, in: Pyke, F; Becattini, G.; Sengenberger, W. (eds.), Industrial districts and inter-firm co-operation in Italy, International Institute of Labour Studies, Geneva 1990

- Carbonara, N., Information and communication technology and geographical clusters, Technovation, Vol. 25, No 3, 2005

- Carbonara, N., New models of inter-firm networks within industrial districts, Entrepreneurship \& Regional Development, Vol. 14, No 3, 2002

- Chiarvesio, M.; Di Maria, E.; Micelli, S., From local networks of SMEs to virtual districts? Evidence from recent trends in Italy, Research Policy, Vol. 33, N 10, 2004

- Chung, S.; Kim, G. M., Performance effects of partnership between manufacturers and suppliers for new product development: The supplier's standpoint, Research Policy, Vol. 32, No 4, 2003

- Chung, S., Building a national innovation system through regional innovation systems, Technovation, Vol. 22, No 8, 2002

- Corolleur, F.; Courlet, C., The Marshallian Industrial District, an organizational and institutional answer to uncertainty, Entrepreneurship \& Regional Development, Vol. 15, No 4, 2003

- Day, M.; Burnett, J.; Forrester, P.L.; Hassard, J., Britain's last industrial district? A case study of ceramics production, International Journal of Production Economics, Vol. 65, No 1, 2000

- Dosi, G.; Freeman, C.; Nelson, R.; Silverberg, G.; Soete, L., Technical change and economic theory, Pinter Publishers, London 1988

- Freel, M. S., Sectoral patterns of small firm innovation, networking and proximity, Research Policy, Vol. 32, No 5, 2003

- Freeman, C.; Soete, L., The economics of industrial innovation, $3^{\text {a }}$ ed, Pinter, 1997

- Freeman, C., Technology and Economic Performance: Lessons from Japan, Pinter, London 1987

- Giner, J. M.; Santa Maria, M. J., Territorial systems of small firms in Spain: an analysis of productive and organizational characteristics in industrial districts, Entrepreneurship \& Regional Development, Vol. 14, No 3, 2002

- Guerrieri, P.; Pietrobelli, C., Industrial districts' evolution and technological regimes: Italy and Taiwan, Technovation, Vol. 24, No 11, 2004

- Kline, S. J.; Rosenberg, N., An overview of innovation, in Landau, R.; Rosenberg, N. (eds.), The positive sum strategy - Harnessing technology for economic growth, The National Academy Press, Washington 1986 
- Lazerson, M.; Lorenzoni, G., Resisting organizational inertia: The evolution of industrial districts, Journal of Management and Governance, Vol. 3, No 4), 1999

- Lundvall, B.-A., National Systems of Innovation - Towards a theory of innovation and interactive learning, Pinter, London 1992

- Marshall, A., Princípios de Economia, Vol.I, tradução revista de Rómulo de Almeida e Ottolmy

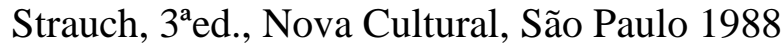

- Molina-Morales, F. X., The territorial agglomerations of firms: A social capital perspective from the Spanish tile industry, Growth and Change, Vol. 36, No 1, 2005

- Molina-Morales, F. X., Industrial districts and innovation: The case of the Spanish ceramic tiles industry, Entrepreneurship \& Regional Development, Vol. 14, N 4, 2002

- Molina-Morales, F. X.; Martínez-Fernández, M. T., Factors that identify industrial districts: An application in Spanish manufacturing firms, Environment and Planning, Vol. 36, No , 2004a

- Molina-Morales, F. X.; Martínez-Fernández, M. T., How much difference is there between industrial district firms? A net value creation approach, Research Policy, Vol. 33, No 3, 2004b

- Muller, E.; Zenker A., Business services as actors of knowledge transformation: The role of KIBS in regional and national innovation systems, Research Policy, Vol. 30, Nº 9, 2001

- Muscio, A., From regional innovation systems to local innovation systems: Evidence from Italian industrial districts, European Planning Studies, Vol. 14, Nº 6, 2006

- Nelson, R., National innovation systems: A comparative analysis, Oxford University Press, 1993

- OECD, Oslo Manual, 1997

- Piore, M. J., Work, labour and action: Work experience in a system of flexible production, in: Pyke, F; Becattini, G.; Sengenberger, W. (eds.), Industrial districts and inter-firm co-operation in Italy, International Institute of Labour Studies, Geneva 1990

- Piore, M. J.; Sabel, C. F., The second industrial divide, Basic Books, New York 1984

- Piscitello, L.; Sgobbi, F., Globalisation, E-Business and SMEs: Evidence from the italian district of Prato, Small Business Economics, Vol. 22, No 5, 2004

- Pyke, F.; Sengenberger, W., Industrial districts and local economic regeneration: Research and policy issues in: Pyke, F.; Sengenberger, W. (eds.), Industrial districts and local economic regeneration, International Institute of Labour Studies, Geneva 1992

- Pyke, F.; Sengenberger, W., Introduction in: Pyke, F.; Becattini, G.; Sengenberger, W. (eds.), Industrial districts and inter-firm co-operation in Italy, International Institute of Labour Studies, Geneva 1990

- Porter, M., Clusters and the new economics of competition, Harvard Bussiness Review, Vol. 76, $\mathrm{N}^{\circ} 6,1998$

- Porter, M., A vantagem competitiva das nações, tradução de Wattensir Dutra, Editora Campus, 1993

- Rabellotti, R., Is there an "industrial district model"? Footwear districts in Italy and Mexico compared, World Development, Vol. 23, Nº 1, 1995

- Sammarra, A.; Biggiero, L., Identity and identification in industrial districts, Journal of Management and Governance, Vol. 5, N ${ }^{\circ}$ 1, 2001

- Sousa, A., Sistema Português da Inovação - Imagem para um enquadramento político, FEP, Universidade do Porto, Porto 1997

- Staber, U., The structure of networks in industrial districts, International Journal of Urban and Regional Research, Vol. 25, No 3, 2001a

- Staber, U., Spatial proximity and firm survival in a declining industrial district: The case of knitwear firms in Baden-Wurttemberg, Regional Studies, Vol. 35, N 4, 2001b

- Teece, D. J., Capturing value from technological innovation: Integration, strategic partnering, and licensing decisions, in: Teece, D. J., Strategy, technology and public policy, Edward Elgar Publishing, 1997 